\title{
Purposeful physical activity-Basic strategy for the prophylaxis and treatment of diabetes and diabetic polyneuropathy.
}

\author{
Petya Kasnakova $^{1}$, Anna Mihaylova1 ${ }^{1}$, Penka Petleshkova ${ }^{2 *}$, Nikoleta Traykova ${ }^{3}$, Biyanka Tornyova ${ }^{4}$ \\ ${ }^{1}$ Medical College, Medical University of Plovdiv, Bulgaria \\ ${ }^{2}$ Department of Obstetrics and Gynecology, Medical University of Plovdiv, Bulgaria \\ ${ }^{3}$ Department of Radiology, UMHAT, “St. George” Plovdiv, Bulgaria \\ ${ }^{4}$ Department of Public Health, Medical University Plovdiv, Bulgaria
}

\begin{abstract}
Diabetes mellitus type $\mathbf{2}$ is a socially significant disease due to its high incidence rate on a global scale. The causes are rooted mainly in the changes in eating habits, the sedentary way of life, the low energy expenditure leading to obesity, the ageing population, as well as urbanization. Diabetes mellitus type $\mathbf{2}$ is a chronic, lifelong condition that requires continuous complex health care and the motivated involvement of a well-trained patient in order for the risk of long-term complications to be reduced. The first step in the treatment entails a change in the way of life. The most significant preconditions in the strategy for prophylaxis and treatment of diabetes mellitus type 2 and the long-term complications include systematic medicamentous therapy in combination with a good control of the condition, physical rehabilitation and physiotherapy with an active exercise routine, strict control of the body weight and healthy eating habits through a balanced diet, as well as limiting any bad habits for the purpose of optimizing the patient's way of life. The aim of the primary and secondary physiotherapeutic prophylaxis is the strengthening of the organism achieved through adequate physical exercise, sports, heliotherapy and air procedures, aquatic therapy and balneotherapy. The medical and social impact of diabetic polyneuropathy and the effect of exercise on the condition call for basic physical rehabilitation routine designed to improve the quality of life of patients with diabetes mellitus.
\end{abstract}

Keywords: Prophylaxis, Treatment, Diabetes mellitus type 2, Physical rehabilitation, Physiotherapeutic prophylaxis. Accepted on March 15, 2019

\section{Introduction}

Type 2 diabetes mellitus (T2DM) is one of the commonest forms of chronic disease globally and few societies or ethnic spared. It accounts for about $85 \%$ of cases of diabetes in white Europeans and virtually all other ethnic groups. In 2015, the International Diabetes Federation (IDF) estimated that 415 million people worldwide had diabetes, of whom $75 \%$ live in low- and middle-income countries. Among those aged 20-79 years, about $8.8 \%$ had diabetes globally, of whom an estimated $46.5 \%$ remain undiagnosed. The highest number of people with diabetes was in the Western Pacific region, with 153.2 million, and there gion with the highest prevalence rate, at $12.9 \%$, was North America and the Caribbean [1,2]. The number of people with diabetes is expected to reach 642 million by 2040, an increase of $55 \%$ [1]. Now-a-days, increased morbidity is associated with the so called "Western" way of life characterized by inadequate physical activity and obesity [3].

There has been an increase in the number of children and adolescents diagnosed with diabetes mellitus type 2, with obesity being the main cause. This is the case with $80 \%$ of the patients with diabetes mellitus type 2 [4]. The "Diabetes epidemics" is highly likely to continue spreading, if obesity levels remain constant. In view of the increasing number of obesity cases, these projections may well prove to be below the actual figures for the future incidence of diabetes [5].

A similar trend has been observed in Bulgaria, too. In 2014, over 577,000 people had diabetes. $10 \%$ of them had diabetes type 1 . Half of the patients with diabetes 220,000 were in prediabetic condition, and about 170,000 were undiagnosed cases of the disorder. Virtually, it means that diagnosed with diabetes (actually registered in the healthcare system) in the country were 140,000 people, or $2 \%$ of the population. It is projected that by 2030 the number of patients with diabetes will have increased by $54 \%$ [6].

The biggest socially significant challenge posed by diabetes mellitus involves the subsequent complications. Poor glycaemic control is the leading factor for the development of the main complications: microangiopathy, macroangiopathy, 
retinopathy, and nephropathy, typical of type diabetes mellitus. Nephropathy is also prevalent in patients with diabetes mellitus type 2 the incidence ranges from $10 \%$ to $50 \%$ depending on how long the patients have had the condition [7]. Diabetic foot is also a common complication in patients with diabetes mellitus, which may result in amputation, permanent disability and deteriorated quality of life. All these complications lead to severe consequences both for the patient and his/her family, on the one hand, and for society as a whole, on the other.

Cardiovascular complications, which occur earlier and progress quickly, constitute the main cause of death in cases of diabetes mellitus. The leading cause for these complications is the development of an arteriosclerotic process mainly due to dyslipidemia, hypertension and hyperglycemia. Diabetes mellitus type 2 is part of the so called metabolic syndrome, which is characterized by the build-up of risk factors for death related to cardiovascular problems [8].

Early prophylaxis implemented as early as the stage of disturbed carbohydrate tolerance, or, on the basis of combined criteria, to overweight people with a family history of the condition, with a sedentary way of life, stressful life experiences, has a significant effect on the subjective complaints and objective metabolic indicators [9].

The main therapeutic approach developed using medical and social rehabilitation is aimed at mitigating the risk of long-term complications that diabetes mellitus entails. The adequate treatment is an integrated, multi-factor one, and is most efficient if implemented in the early stages of the conditionprior to the onset of complications [10].

\section{Literature Review}

According to the standards of the American Diabetes Association (ADA), the European Diabetes Policy Group and the World Health Organization, the monitoring of patients with diabetes mellitus and diabetic polyneuropathy has to be conducted on a regular basis by the so called 'diabetic team' of various specialists.

The most efficient approach to solving the problems of diabetics is the interdisciplinary one. The treatment of patients with diabetes always has to be integrated, both targeted at all the risk factors and components of complications, on the one hand, and consistent with the rest of the somatic pathology, in standby for intensive medical care. The team of specialists, as recommended by the World Health Organization, should include an endocrinologist-diabetologist, a vascular surgeon, a radiographer, an orthopedist, a neurologist, a nurse, a chiropodist, an orthotist, a physical rehabilitation specialist, a psychotherapist, and social workers.

Numerous studies show that the integrated approach, early diagnostics, the training of the patient, may significantly reduce the number of patients with complications [11]. The training has to be targeted not only at the patient but also at his/her family and the whole team of healthcare providers. The aim is not only to raise the patient's awareness but also to enhance his/her motivation to achieve self-management and self-control through specific habits and skills [10].

The multi-factor health care has to be focused on primary care. Primary care should, first and foremost, include screening, identification of the risk factors, diagnostics, and the development of a training program for working with the patient. Giving up smoking, making positive changes to the way of life, adequate nutrition, physical activity with targeted exercise programs are key prophylactic measures [12].

Diabetic neuropathy is a leading pathogenic factor for the onset of diabetes-related complications affecting the lower extremities-diabetic foot, most commonly characterized by the loss of sensitivity, an increased risk of ulcerations and amputation.

\section{Therapeutic Approaches}

The common strategies for managing diabetes mellitus generally include pharmacological and non-pharmacological treatment. The pharmacological treatment can be pathogenic and symptomatic. The non-pharmacological treatment includes integrated rehabilitation programs including kinesitherapy, preformed physical factors, balneotherapy, and climatic treatment. The best practices include dietary measures for a strict control of the blood sugar level, a balanced diet and control of the body weight, an active exercise routine and reducing the consumption of alcohol to an absolute minimum.

The pharmacological treatment of polyneuropathy is recommended to include different medication groups; vitamins of the group BB1, B6, B12, alpha-lipoic acid, gamma-linolenic acid, which improves the nerve conduction, myotropic drugs with a direct vasodilatory effect, antiaggregants, vasodilators, neuromediators, etc. [12-14].

Patients (middle-aged and elderly ones, in particular) have to change their way of life and start doing moderate but regular exercise so as to maintain a certain level of physical fitness. Physical activity reduces the insulin resistance of the skeletal muscles, prevents from obesity, helps reduce body weight, which is associated with lower plasma levels of LDLcholesterol and triglycerides, and higher plasma levels of HDL-cholesterol, as well as with lower blood pressure.

Kinesitherapy is one of the main approaches to treating diabetic polyneuropathy, and may even counteract the risk factors for the onset and progress of the condition [15]. Regular physical activity aims to maintain the muscles in good condition, to provide prophylaxis against inactive hypotrophy and contractures, to maintain the emotional status of the patient, to improve the social adaptability and overall vitality [16]. The active kinesitherapy program prevents the occurrence of the extremely dangerous "ping-pong" effects of medication on blood-sugar levels, and reduces the extreme glycemic levels, which are really challenging to patients with diabetes [17]. This makes kinesitherapy a powerful ally of modern antidiabetic medication (sulfonylureas, acarbose, insulins). The following are recommended: generally strengthening exercise, 
analytical and functional exercise, stretching, passive mobilization, remedial exercise, non-competitive games, field exercise, tourism and physical exercise as prescribed, walking, swimming, cycling of moderate intensity (upto $60 \mathrm{~W}$ ), and a duration of 2-3 hours. Longer physical exercise leads to a significant decrease of the blood-sugar level. Contraindicative are exercises causing quick changes in the position of the body, intensive short-term physical exercise of over 100-200 W (running and weight-lifting), as these pose a risk of severe hypoglycemia. Exercises with an inflatable soft ball, with elements of tai-chi, yoga and gym exercises, underwater exercise medicinal massage in the form of a massage collar or general massage, are more commonly used as means to counteract the hypodynamia of the modern man [18]. Kinesitherapy is usually recommended in the form of walks, cross-country hiking or walking along the beach for 3 to 5 hours a day. The physical strain is slight to moderate (3.5 to 5 $\mathrm{km} / \mathrm{h}$ ), and a heartbeat of 90 to 110 beats $/ \mathrm{min}$.

When doing exercise, patients with diabetes have to bear in mind the following specifics: only light to moderately straining exercise should be done, more intensive exercise poses the risk of ketoacidosis and vascular damage; for patients with vascular degenerative changes it is recommended that a preliminary assessment of the capacity of the cardiovascular system should be made. It is of no importance what kind of exercise that is to be done is of no importance as an increased breakdown of sugar is observed in both the activated muscles and the nonengaged ones. Glucose utilization is most expressed in cases of exercise done in cool and fresh thermo-meteorological conditions. As a rule, exercise in conditions of cold or heat stress should be avoided. Physical exercise reduces the need of insulin. Conditions for reducing the needs of insulin, and reducing or eliminating the intake of perorally administered antidiabetic medication. Regular physical exercise reduces hypercholesterolemia and hypertriglyceridemia [15]. Due to the risk of hypoglycemia during physical exercise, the food portions should be strictly distributed and the insulin doses should be adjusted. A moderately reducing balanced diet is recommended-mainly vegetables, non-sweet fruit, dairy products and meat protein. Carbohydrates should be between 120 to $300 \mathrm{~g}$ per day, protein-100-120 g, and fat-50-60 g.

Patients with diabetes type 2 often suffer from comorbidities and are often overweight, therefore physical exercise should be scheduled individually, and should be consistent with and appropriate for the overall status of the patient. Physical exercise is indicated for patients with blood sugar levels between 3.5 and $8 \mathrm{mmol} / \mathrm{l}$. Ergotherapy can successfully be applied in the form of gardening, fruit-growing, horticulture, and other everyday activities, depending on the climatic conditions.

Preformed physical factors are also applied in cases of diabetic polyneuropathy for the purpose of improving circulation, tissue trophicity and nerve conduction. Electro and light therapy in patients with diabetes mellitus and diabetic polyneuropathy are aimed at enhancing the defense and adaptation capacity of the organism, and improving the supply of oxygen to the tissues.
Electrophoresis with nivalin, ultraviolet radiation therapy and low-frequency impulse magnetic field, as well as laser therapy is administered [17].

For achieving a more beneficial effect, kinesitherapy is combined with field therapy and climatotherapy. All forms of air therapy are very often used: day, night, whole-day sleep in the open, or air baths in fresh and cool meteorological conditions (the sub-comfort zone), with a duration of 2-3 hours a day. This therapy is accepted as a natural oxygen therapy, and conditions for a more beneficial supply of oxygen to the tissues are created [19].

As for balneotherapy, the focus is on the external application of various types of mineral water. Baths in radon mineral water with a radon concentration of $1.5-3 \mathrm{kBq} / 1$, carbonic acid baths with a $\mathrm{CO}_{2}$ concentration of up to $2 \mathrm{~g} / \mathrm{l}$ or sulfide mineral water baths with a hydrogen sulfide content of 50-60 mg/l are recommended. The most beneficial for drinking is alkaline hydrocarbonic sulfate mineral water. Medicinal mud and lye therapy are also applied in the form of compresses [19].

\section{Discussion}

The regular implementation of physical rehabilitation programs in cases of diabetes mellitus type 2 and diabetic neuropathy is considered a targeted, efficient therapy, with a physiological mechanism of action. The utilization of glucose by the muscles is improved, lipolysis is triggered in the fatty tissues, and the body weight is reduced in obese patients. The patients'quality of life is improved and a significant effect is achieved against the main risk factors (insulin resistance, weight, dyslipidemia, etc.), the defense capacity of the organism is stimulated [20].

The prophylaxis of diabetes constitutes health promotion aimed at raising the awareness of the population of the disorder, the risk factors and possible complications. So far, there has been no established prophylaxis for diabetes mellitus type 1 , and the prophylaxis of obesity has an impact on type 2 [20]. Primary prophylaxis involves developing appropriate eating habits and counteracting obesity as a risk factor. Secondary prophylaxis is aimed at preventing complications: the early detection using screening tests, good control of the progress of the condition, intensive therapy through proper diet and medications. Tertiary prophylaxis includes the restoration of the functions of the affected organs and systems, and the prevention of further damage [21]. The main therapeutic approach is focused on reducing the risk of long-term damage through medical and social rehabilitation and reintegration of the patients.

Aerobic, anaerobic, and resistance training exercises can be used alone or in combination to achieve the desired effect of improving cardiorespiratory fitness, muscle strength and endurance, weight loss and its maintenance. The type, intensity and volume of exercise should be tailored to individual needs to allow maximal adherence and long-term health benefits. Although the benefits greatly outweigh the risks, there are restrictions to exercise for people with certain medical 
conditions in terms of the type and intensity of physical activity [22].

There is broad consensus in the global diabetes community that diabetes education and psychosocial support are critical elements of care for all people with diabetes and their family members [23]. The aim of providing diabetes education and psychosocial support is to promote behaviors that will lead to the prevention or delay of the complications of diabetes and to enhance good quality of life [23].

The lifelong process of diabetes self-management requires continued adjustments in knowledge, skills, motivation, and support. Education and support should be a lifelong process, starting at the point of diagnosis and remaining as an essential component of diabetes care [24,25].

\section{Conclusion}

In conclusion, it can be summed up that regular courses of systematically conducted rehabilitation and physical therapy including the use of natural factors (mineral water, peloids, and climate) could significantly enhance the medication therapy of patients with diabetes mellitus and diabetic polyneuropathy, and would improve the quality of life of this group of patients.

People with diabetes have to live the rest of their lives with the condition and therefore need to improve their knowledge, skills, and confidence, enabling them to take better control of their own condition through the positive improvement of their beliefs. They also have to integrate effective self-management into their daily lives. These aims can be achieved through structured patient education programs.

\section{References}

1. International Diabetes Federation. IDF Diabetes Atlas, 7th edition. Brussels: International Diabetes Federation 2015.

2. Shaw JE, Sicree RA, Zimmet PZ. Global estimates of the prevalence of diabetes for 2010 and 2030. Diabetes Res Clin 2010; 87: 4-14.

3. Tan GH, Nelson RL. Pharmacologic treatment options for non-insulin-dependent diabetes mellitus. Mayo Clin Proc 1996; 71: 763-768.

4. Barrett T. Type 2 diabetes mellitus: incidence, management and prognosis Paediatr Child Health 2013; 27: 163-167.

5. The International Diabetes Federation (IDF) card for the global prognosis for the number of people with diabetes in 2010 and 2030. Club D Journal, Specialized Diabetes Edition, in line of Dr. D. Yankova and Dr. L. Goranova, Sofia, November 2013, issue 5, 89.

6. Estimates of Type 1 Diabetes in Bulgaria for $2030 \mathrm{http}: / /$ light.standartnews.com/mneniya-intervyuta/

13_ot_uchenitsite_s_nadnormeno_teglo_-263967.html

7. Boulton AJM, Vinik AI, Arezzo JC, Bril V, Feldman EL, RoFreeman YMB, Malik RA, Maser RE, Sosenko JM, Ziegler D. Diabetes Neuropathies. A statement by the American Diabetes Association. Diabetes Care 2005.
8. American Diabetes Association. Standards of medical care in diabetes - 2013. Diabetes Care 2013; S11-S66.

9. Berry D, Melkus GD. Epidemiologic perspectives of risk for developing diabetes and diabetes complications. Nurs Clin Norht Am 2006; 41: 487-498.

10. Bochcelian H. Diabetic foot. A brief guide on diagnosis and treatment. Gea print, Varna, 2006.

11. Stambolova I, Terzieva A, Vassileva N. Collaboration and patient involvement in the therapeutic team. Health Care, 2011, No 2, 24-28.

12. Botushanov N. Diabetic neuropathy. Doctor Practice 2003; 36-42.

13. Wild S, Roglic G, Green A, Sicree R, King H. Global prevalence of diabetes: Estimates for the year 2000 and projections for 2030. Diabetes Care 2004; 27:1047-1053.

14. Bennett WL, Maruthur NM, Singh S, Segal JB, Wilson LM, Chatterjee R, Marinopoulos SS, Puhan MA, Ranasinghe P, Block L, Nicholson WK, Hutfless S, Bass EB, Bolen S. Comparative effectiveness and safety of medications for type 2 diabetes: An update including new drugs and 2-drug combinations. Ann Intern Med 2011; 154:602-613.

15. Lubenova E. Practical guidelines for the use of specialized kinesitherapy in diabetic polyneuropathy. Coti EOOD, Sofia, 2008.

16. Gomez J. Living with Diabetis, Sheldon Press Ltd 2002.

17. Koleva I. Algorithms for physical prevention, therapy and rehabilitation of some common and socially significant diseases. Sofia 2007.

18. Zhelev V. Physiotherapy, third part. Specialized Methods and Methods in Physiotherapy. Sofia 2011.

19. Edreva V, Krasteva D. Balneotherapy, water therapy, mud treatment. Ivay Sofia 2000.

20. Borisova AM. Recommendations for good clinical practice in diabetes mellitus. Bulgarian Society of Endocrinology. Sofia 2013.

21. Bloomgarden ZT. American diabetes association 60th scientific sessions, 2000: The diabetic foot. Diabetes Care 2001; 24: 946-951.

22. Chen M, Andrews R. Life style Issues: Exercise. Textbook of Diabetes, Fifth Edition. Edited by Holt R, Cockram C, Flyvbjerg A, Goldstein B 2017; 354.

23. Haas L, Maryniuk M, Beck J, Cox CE, Duker P, Edwards L, Fisher EB, Hanson L, Kent D, Kolb L, McLaughlin S, Orzeck E, Piette JD, Rhinehart AS, Rothman R, Sklaroff S, Tomky D, Youssef G, 2012 Standards Revision Task Force. Diabetes Care 2014; 37: S144-153.

24. Willaing L, Vallis M. Educating the person with diabetes. Textbook of Diabetes, Fifth Edition. Edited by Holt R, Cockram C, Flyvbjerg A, Goldstein B 2017; 338.

25. Davies MJ, Heller S, Skinner TC, Campbell MJ, Carey ME, Cradock S, Dallosso HM, Daly H, Doherty Y, Eaton S, Fox C, Oliver L, Rantell K, Rayman G, Khunti K, Diabetes education and self-management for ongoing and newly diagnosed collaborative. Effectiveness of the diabetes education and self-management for ongoing and 
Purposeful physical activity-Basic strategy for the prophylaxis and treatment of diabetes and diabetic polyneuropathy.

newly diagnosed (DESMOND) programme for people with newly diagnosed type 2 diabetes: Cluster randomised controlled trial. BMJ 2008; 336: 491-495.

\section{*Correspondence to:}

Penka Petleshkova

Department of Obstetrics and Gynecology

Medical University of Plovdiv

Bulgaria 